Rev. Bras. Saúde Prod. Anim., Salvador, v.16, n.1, p.47-56 jan./mar., $2015 \quad \underline{\text { http://www.rbspa.ufba.br }}$ ISSN 15199940 http://dx.doi.org/10.1590/S1519-99402015000100006

\title{
Grupo genético e ordem de parto no desempenho produtivo e reprodutivo de matrizes suínas
}

Genetic group and parity order on productive and reproductive performance of sows

\author{
ROSA, Lorena Silva da ${ }^{1 *}$; SOUZA, Maria Inês Lenz ${ }^{2}$; CORREA FILHO, Ruy Alberto \\ Caetano $^{3}$; COSTA FILHO, Luiz Carlos Cesar da ${ }^{1}$
}

\begin{abstract}
${ }^{1}$ Universidade Federal de Mato Grosso do Sul, Faculdade de Medicina Veterinária e Zootecnia, Programa de Pós-Graduação em Ciência Animal, Campo Grande, Mato Grosso do Sul, Brasil.

${ }^{2}$ Universidade Federal de Mato Grosso do Sul, Centro de Ciências Biológicas e da Saúde,

${ }^{3}$ Universidade Federal de Mato Grosso do Sul, Faculdade de Medicina Veterinária e Zootecnia

*Endereço para correspondência: lorenarosa.vet@ hotmail.com
\end{abstract}

\section{RESUMO}

O objetivo com este trabalho foi avaliar o efeito de grupo genético (GGen) e de ordem de parto (OrdP) de 400 matrizes suínas sobre características produtivas e reprodutivas. Utilizouse três GGen (GGen1, GGen2 e GGen3) e duas OrdP (OrdP1 e OrdP2). As variáveis dependentes avaliadas foram peso médio da leitegada ao nascimento (PMNasc), peso médio da leitegada ao desmame (PMDesm), tamanho da leitegada ao nascimento (TNasc), tamanho da leitegada ao desmame (TDesm), duração da lactação (DL), número de partos/fêmea/ano (P/F/A), número de leitões desmamados/fêmea/ano (D/F/A), ganho de peso diário do leitão (GPD), ganho de peso diário da leitegada (GPDLeit), intervalo desmame-cio (IDC), média de dias não produtivos (MDNP) e número de serviços por concepção (NSPC). A interação entre GGene OrdPfoi significativa para PMDesm, DL, P/F/A, GPD, IDC e MDNP. Para as demais variáveis (PMNasc, TNasc, TDesm e D/F/A) não ocorreu interação nem efeito de GGen e OrdP. Houve efeito do GGen independente da OrdP nas variáveis GPDLeit e do NSPC. O GGen 3 foi considerado superior, uma vez que este apresentou melhor desempenho em relação aos demais na maioria das características avaliadas. Não foi possível determinar a melhor OrdP, pois esta variou em função dos GGen e das variáveis dependentes.

Palavras-chave: banco de dados, matrizes cruzadas, ordem de parto

\section{SUMMARY}

The objective of this paper was toevaluate the effect of genetic group (GG) and parity order (PO) of 400 sows on productive and reproductive traits. Were used three GG (GG1, GG2 and GG3) and two PO (PO1 and PO2). The dependent variables evaluated were litter weight at birth (LWB), litter weight at weaning (LWW), litter size at birth (LSB), litter size at weaning (LSW), lactation length (LL), number of litters/female / year $(\mathrm{L} / \mathrm{F} / \mathrm{Y})$, number of piglets weaned / female / year (P/W/F), daily weight gain of each piglet (DWG), average daily gain of piglets (ADGP), weaning to estrus interval (WEI), average non-productive days (ANP) and number of services per conception (NSPC). The interaction between GG and $\mathrm{PO}$ was significant for $\mathrm{LWW}, \mathrm{LL}, \mathrm{L} / \mathrm{F} / \mathrm{Y}$, DWG, WEI e ANP. For the other variables (LWB, LSB, LSW and P/W/F) no interaction or effect of GG and PO was observed. There was effect of GG independent of PO in the variables except ADGP and NSPC. The GG 3 was considered superior, since it showed better performance in relation to others in most of characteristics evaluated. It was not possible to determine the best PO, because this varied according to GG and the dependents variables.

Keywords: crossbred sows, database, parity order 
Rev. Bras. Saúde Prod. Anim., Salvador, v.16, n.1, p.47-56 jan./mar., 2015 http://www.rbspa.ufba.br ISSN 15199940 http://dx.doi.org/10.1590/S1519-99402015000100006

\section{INTRODUÇÃO}

O Brasil é o quarto maior produtor de carne suína do mundo, sendo precedido pela China, União Europeia e Estados Unidos, além de possuir, atualmente, o terceiro maior rebanho mundial, com mais de 39 milhões de cabeças (IBGE, 2011). No primeiro semestre de 2013, as exportações brasileiras de carne suína atingiram aproximadamente 291 mil toneladas, contabilizando (uma cifra US\$ 756 milhões).

No Brasil, o melhoramento genético suíno iniciou-se na década de 70, com a importação de animais da Europa e América do Norte. A seleção hoje praticada, nos rebanhos núcleo de matrizes, além de considerar os parâmetros de desempenho, como o ganho de peso diário, fundamenta-se em características reprodutivas, principalmente nonúmero de leitões nascidos vivos e desmamados, e no peso da leitegada ao desmame. O desempenho reprodutivo do rebanho brasileiro, graças à introdução de material genético melhorador e dos programas de melhoramento que foram estabelecidos no país, não deixa adesejar quando comparado ao de outros países com suinocultura de alta tecnologia (FÁVERO \& FIGUEIREDO, 2009).

$\mathrm{O}$ conhecimento dos fatores que influenciam as características produtivas e reprodutivas em suínos torna-se cada vez mais relevante, dada à diversidade geográfica brasileira, manejos deficientes, instalações inadequadas e animais que, nem sempre, são de alto valor genético (PINHEIRO et al., 2000). Dentre eles, podemos citar os fatores genéticos $\mathrm{e}$ ambientais (PIRES et al, 2008).

A ordem de parição da matriz exerce significativa influência na fertilidade da mesma, sendo que a eficiência reprodutiva da fêmea aumenta ao longo da idade e, posteriormente, declina nas ordens de parto mais avançadas (MELLAGI et al., 2013). Essas diferenças foram encontradas na taxa de parto (VARGAS et al., 2009), no tamanho da leitegada no desmame e no intervalo desmame-cio (KOKETSU \& Dial, 1997).

Um constante acompanhamento das características economicamente importantes faz-se necessário em cada grupo genético e sua relação com a ordem de parto das fêmeas suínas, para que, assim, possam ser planejados os melhores cruzamentos. $\mathrm{O}$ objetivo deste trabalho foi avaliar três grupos genéticos e duas ordens de parto em relação às características de desempenho produtivo e reprodutivo de matrizes suínas comerciais.

\section{MATERIAL E MÉTODOS}

Utilizou-se o banco de dados de uma Unidade Produtora de Leitões (UPL II), situada no município de São Gabriel do Oeste (latitude $19^{\circ} 23^{\prime} \mathrm{S}$, longitude $54^{\circ} 23^{\prime} \mathrm{W}$ ), vinculada a um sistema de propriedades suinícolas parceiras da Cooperativa Agrícola São Gabriel do Oeste - COOASGO. A granja dispunha de um plantel de 927 matrizescriadas em confinamento, provenientes de diferentes linhagens genéticas, sendo 856porcas e 71 leitoas, em que todos os animais da propriedade encontravam-se sob a mesma dieta alimentar e mesmo manejo de produção.

Foram selecionadas, aleatoriamente, 400 fêmeas para avaliação dos registros individuais de desempenho, no período de março de 2011 a março de 2013. Buscaram-se relatórios referentes à 
Rev. Bras. Saúde Prod. Anim., Salvador, v.16, n.1, p.47-56 jan./mar., 2015 http://www.rbspa.ufba.br ISSN 15199940

reprodução de fêmeas de três grupos genéticos (GGen1, GGen2 e GGen3), sendo o GGen1 ( $\mathrm{n}=46$ matrizes) oriundo do cruzamento entre as raças Large White $\mathrm{x}$ Landrace x Duroc e, os GGen2 ( $\mathrm{n}=124$ matrizes) e GGen3 ( $n=230$ matrizes), ambos resultantes do cruzamento entre as raças Large White x Landrace, porém provenientes de diferentes empresas. Todos os animais apresentaram ciclo reprodutivo até o segundo parto (ordem de parto 1 - OrdP1, ordem de parto 2 OrdP2). No período da coleta de dados, o controle de produção na granja foi efetuado com o auxílio do programa de gerenciamento de granjas de suínos Agriness ${ }^{\circledR}$ S2. Este programa gera relatórios retrospectivos contendo os dados de desempenho acumulados ao longo da vida reprodutiva das matrizes, de onde foram retiradas as informações para análises deste estudo.

Como variáveis dependentes analisaramse peso médio da leitegada ao nascimento (PMNasc), peso médio da leitegada ao desmame (PMDesm), tamanho da leitegada ao nascimento (TNasc) o qual foi adotado, tamanho da leitegada ao desmame (TDesm), duração da lactação (DL), número de partos/fêmea/ano (P/F/A), número de leitões desmamados/fêmea/ano (D/F/A), ganho médio de peso diário do leitão (GPD), ganho médio de peso diário da leitegada (GPDLeit), intervalo desmame-cio (IDC), média de dias não produtivos das matrizes (MDNP) e número de serviços por concepção (NSPC).

Os dados foram submetidos a uma análise exploratória prévia e as informações inconsistentes (outliers) eliminadas. O tamanho da leitegada ao nascimento foi determinado pela somatória do número de leitões nascidos vivos, natimortos $\mathrm{e}$ mumificados. As variáveis peso médio da leitegada ao nascimento e tamanho da leitegada ao nascimento foram obtidas 24 horas após o nascimento, depois do manejo de reagrupamento e da homogeneização da leitegada. O desmame foi realizado, entre 3-4 semanas após o parto. Como critério de exclusão, eliminaram-se do estudo matrizes com dias não produtivossuperioresa 40 dias, determinados conforme a fórmula (365 [PARTOS/F/A X (dias gestante - dias lactante), pois acredita-se que possa ter havido do erro de manejo ou de anotação de dados pelos funcionários da granja.

As análises estatísticas foram realizadas com o auxílio do programa computacional SAS 9.0 (STATISTICAL ANALYSIS SYSTEMS, 2004), utilizando o procedimento GLM para análise de variância, com posterior teste de Tukey-Kramer, no nível de 5\% de probabilidade. $\mathrm{O}$ modelo utilizado incluiu o efeito de grupo genético, de ordem de parto e a interação entre estes efeitos. As variáveis que não atenderam às exigências dos testes paramétricos foram analisadas pelo teste de Kruskal-Wallis, com posterior teste de Dunn, no nível de probabilidade de 5\%, com o mesmo modelo anterior e seguindo as recomendações de ZAR (2010).

\section{RESULTADOS E DISCUSSÃO}

A interação entre grupos genéticos e ordens de parto foi significativa para PMDesm, DL, P/F/A, GPD, IDC e MDNP (Tabela 1). Nas variáveis GPDLeit e NSPC o efeito principal de grupo genético foi significativo. Para as demais variáveis PMNasc, TNasc, TDesm e D/F/A não ocorreu interação nem efeito principal de grupo genético e de ordem de parto da matriz suína. 
Tabela 1. Médias de mínimos quadrados ou geométricas para diferentes características reprodutivas de 400 matrizes suínas em função do grupo genético (GGen1, GGen2, GGen3) e da ordem de parto (OrdP1, OrdP2), de um estudo conduzido entre 2011 e 2013, no Estado de Mato Grosso do Sul

\begin{tabular}{|c|c|c|c|c|c|c|c|c|c|c|c|}
\hline $\mathrm{PMNasc}^{\mathrm{I}}$ & & OrdP & & PMD & & OrdP & & TNasc & & OrdP & \\
\hline GGen & 1 & 2 & Total & GGen & 1 & 2 & Total & GGen & 1 & 2 & Total \\
\hline 1 & 1,53 & 1,58 & $1,56^{\mathrm{a}}$ & 1 & $6,98^{\mathrm{Aa}}$ & $6,13^{\mathrm{Bb}}$ & - & 1 & 12,42 & 11,17 & $11,79^{\mathrm{a}}$ \\
\hline 2 & 1,50 & 1,53 & $1,51^{\mathrm{a}}$ & 2 & $6,80^{\mathrm{Ba}}$ & $7,44^{\mathrm{Aa}}$ & - & 2 & 10,73 & 10,58 & $10,65^{\mathrm{a}}$ \\
\hline 3 & 1,48 & 1,53 & $1,51^{\mathrm{a}}$ & 3 & $6,26^{\mathrm{Bb}}$ & $6,76^{\mathrm{Ab}}$ & - & 3 & 11,23 & 11,52 & $11,37^{\mathrm{a}}$ \\
\hline Total & $1,50^{\mathrm{a}}$ & $1,55^{\mathrm{a}}$ & - & Total & - & - & & Total & $11,46^{\mathrm{A}}$ & $11,09^{A}$ & - \\
\hline TDesm & & OrdP & & DL & & OrdP & & $\mathrm{P} / \mathrm{F} / \mathrm{A}$ & & OrdP & \\
\hline GGen & 1 & 2 & Total & GGen & 1 & 2 & Total & GGen & 1 & 2 & Total \\
\hline 1 & 10,27 & 9,83 & $10,05^{\mathrm{a}}$ & 1 & $26,27^{\mathrm{Bb}}$ & $21,08^{\mathrm{Aa}}$ & - & 1 & $2,30^{\mathrm{Bb}}$ & $2,45^{\mathrm{Aa}}$ & - \\
\hline 2 & 10,21 & 10,42 & $10,32^{\mathrm{a}}$ & 2 & $25,15^{\mathrm{Ab}}$ & $29,44^{\mathrm{Bb}}$ & - & 2 & $2,43^{\text {Aab }}$ & $2,40^{\mathrm{Aa}}$ & - \\
\hline 3 & 10,53 & 10,38 & $10,46^{\mathrm{a}}$ & 3 & $22,77^{\mathrm{Aa}}$ & $24,07^{\mathrm{Ba}}$ & - & 3 & $2,52^{\mathrm{Aa}}$ & $2,42^{\mathrm{Ba}}$ & - \\
\hline Total & $10,34^{\mathrm{a}}$ & $10,21^{\mathrm{a}}$ & & Total & - & - & & Total & - & - & - \\
\hline $\mathrm{D} / \mathrm{F} / \mathrm{A}$ & & OrdP & & GPD & & OrdP & & GPLeit & & OrdP & \\
\hline GGen & 1 & 2 & Total & GGen & 1 & 2 & Total & GGen & 1 & 2 & Total \\
\hline 1 & 23,98 & 24,33 & $24,16^{\mathrm{a}}$ & 1 & $209,47^{\mathrm{Aa}}$ & $216,56^{\text {Aab }}$ & - & 1 & 2147,02 & 2137,89 & $2142,46^{b}$ \\
\hline 2 & 24,97 & 25,25 & $25,11^{\mathrm{a}}$ & 2 & $214,52^{\mathrm{Aa}}$ & $202,48^{\mathrm{Bb}}$ & - & 2 & 2186,98 & 2119,32 & $2153,15^{b}$ \\
\hline 3 & 25,76 & 25,03 & $25,39^{\mathrm{a}}$ & 3 & $212,12^{\mathrm{Ba}}$ & $220,93^{\mathrm{Aa}}$ & - & 3 & 2227,88 & 2295,04 & $2261,46^{\mathrm{a}}$ \\
\hline Total & $24,90^{\mathrm{a}}$ & $24,87^{\mathrm{a}}$ & & Total & - & - & & Total & $2187,29^{\mathrm{A}}$ & $2184,08^{\mathrm{A}}$ & - \\
\hline $\mathrm{IDC}^{(2)}$ & OrdP & & & MDNP & OrdP & & & NSPC & OrdP & & \\
\hline GGen & 1 & 2 & Total & GGen & 1 & 2 & Total & GGen & 1 & 2 & Total \\
\hline 1 & $6,97^{\mathrm{Aa}}$ & $5,90^{\mathrm{Aa}}$ & - & 1 & $8,4^{\mathrm{Ab}}$ & $8,44^{\mathrm{Aa}}$ & - & 1 & 1,45 & 1,18 & $1,36^{\mathrm{b}}$ \\
\hline 2 & $6,68^{\mathrm{Aa}}$ & $6,19^{\mathrm{Aa}}$ & - & 2 & $7,33^{\mathrm{Ab}}$ & $8,75^{\mathrm{Aa}}$ & - & 2 & 1,50 & 1,34 & $1,45^{\mathrm{b}}$ \\
\hline 3 & $11,2^{\mathrm{Bb}}$ & $4,82^{\mathrm{Aa}}$ & - & 3 & $4,47^{\mathrm{Aa}}$ & $9,53^{\mathrm{Ba}}$ & - & 3 & 1,05 & 1,14 & $1,09^{\mathrm{a}}$ \\
\hline Total & 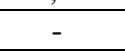 & - & - & Total & - & - & & Total & $1,25^{\mathrm{A}}$ & $1,19^{\mathrm{A}}$ & - \\
\hline
\end{tabular}

${ }^{1}$ PMNasc: peso médio da leitegada 24 horas após o nascimento; PMDesm: peso médio da leitegada ao desmame; TNasc: tamanho da leitegada ao nascimento; TDesm: tamanho da leitegada ao desmame; DL: duração da lactação; P/F/A: partos/fêmea/ano; D/F/A: desmamados/fêmea/ano; GPD: ganho de peso diário; GPDLet: ganho de peso diário da leitegada; IDC: intervalo desmame-cobertura; MDNP: média de dias não produtivos; NSPC: número de serviços por concepção; OrdP: ordem de parto; GGen: grupo genético.

${ }^{2}$ As variáveis IDC, MDNP e NSPC foram submetidas a análises não paramétricas (Teste de Dunn)

Médias de mínimos quadrados ou geométricas seguidas por letras minúsculas distintas nas linhas, em cada variável, diferem entre si pelos Testes de Tukeyou de Dunn $(\mathrm{P}<0,05)$.

Médias de mínimos quadrados ou geométricas seguidas por letras maiúsculas distintas nas colunas, em cada variável, diferem entre si pelos Testes de Tukeyou de Dunn $(\mathrm{P}<0,05)$. 
Após o desdobramento das interações e da aplicação dos testes de comparações de médias ou do teste de Dunn, verificouse influência de grupo genético nas diferentes ordens de parto para PMDesm, DL, P/F/A, GPD, IDC e MDNP, com exceção da variável GPD na primeira ordem de parto e das variáveis P/F/A, IDC e MDNP na segunda ordem de parto. $\mathrm{O}$ efeito simples da ordem de parto foi significativo nos diferentes grupos genéticos para as mesmas variáveis citadas acima, com exceção das variáveis GPD, IDC e MDNP em matrizes do GGen1 e das variáveis P/F/A, IDC e MDNP em fêmeas doGGen2.

$\mathrm{Na}$ OrdP1, matrizes dos GGen1 e GGen2 desmamaram leitegadas mais pesadas que do GGen3, enquanto na OrdP2, o GGen2 obteve desempenho superior para esta característica em relação aos demais. Bianchi et al. (2006) verificaram diferenças entre raças, onde fêmeas de segunda ordem de parto da raça Landrace apresentaram leitegadas com maior peso médio ao desmame que matrizes primíparas da raça Large White. Da mesma forma, Nocera \& Fedalto (2002), encontraram desempenho superior no PMDesm de fêmeas híbridas comerciais (Landrace, Large White e Duroc) em relação às matrizes híbridas da própria granja (Landrace/Large White), ainda que sem influência das seis OrdP avaliadas. No presente estudo, o efeito do manejo adotado na granja pode ter interferido no PMDesm da leitegada, como o manejo de reagrupamento $\mathrm{e}$ homogeneização, além de que as leitegadas provenientes de matrizes do GGen1 e GGen2 foram amamentadas por mais tempo, logo autores afirmam que quanto maior a DL maior é o PMDesm (YANG et al., 2000).

$\mathrm{Na}$ OrdP1, fêmeas do GGen3 foram submetidas a lactações mais curtas que as demais, enquanto na OrdP2, as matrizes dos GGen1 e GGen3 amamentaram durante tempo inferior às porcas do GGen2. Carregaro et al. (2006) afirmam que a variável DL influencia o desempenho reprodutivo subsequente em primíparas e multíparas suínas. Alguns autores sugerem que matrizes de primeira parição ainda não possuem maturidade fisiológica completa (tamanho e reservas corporais), em comparação às multíparas, sendo que o consumo de ração durante o período de lactação é insuficiente para seu desenvolvimento e para as exigências da leitegada (KOKETSU \& DIAL, 1997). Desta forma, há prejuízode seu desempenho reprodutivo posterior. No presente estudo, o efeito do manejo adotado na granja pode ter interferido na DL das fêmeas, de forma que, o período lactacional de algumas matrizes foi mais longo e de outras mais curto, visando o posterior vazio das instalações (vazio sanitário).

$\mathrm{Na}$ OrdP1, as matrizes dos GGen2 e GGen3 tiveram maior número de partos/ano, enquanto na OrdP2, o desempenho para esta característica foi semelhante entre os três GGen avaliados. Waehner \& Bruessow (2009), ao estudarem o potencial de fertilidade de fêmeas Landrace (cruzadas), Large White e Pietrain na Alemanha, encontraram que o melhor desempenho foi obtido em matrizes Landrace cruzadas, com 2,26 partos/ano. No presente estudo, o efeito do manejo adotado na granja pode ter interferido no número de P/F/A de matrizes do GGen3, pois estas amamentaram por períodos mais curtos e, consequentemente produziram maior número de partos/ano.

$\mathrm{Na}$ OrdP1, o GPD foi semelhante para os três grupos genéticos, ao passo que, na OrdP2, os GGen1 e GGen3 produziram leitões com melhor desempenho para esta 
Rev. Bras. Saúde Prod. Anim., Salvador, v.16, n.1, p.47-56 jan./mar., 2015 http://www.rbspa.ufba.br ISSN 15199940

característica. Martins et al. (2007) observaram que a OrdP da matriz influenciou o ganho médio de pesodos leitões, em primíparas e multíparas de segunda e terceira ordens de parição. Entretanto, o grupo genético das primíparas no presente estudo não influenciou o GPD; por outro lado, as multíparas obtiveram leitões com maior GPD em dois grupos genéticos (GGen1 e GGen 3). Neste mesmo sentido, ao avaliarem o desempenho de porcas de primeiro a terceiro parto, Lima et al. (2006) não verificaram efeito significativo da OrdP sobre esta característica.

$\mathrm{Na}$ OrdP1, fêmeas pertencentes aos GGen1 e GGen2 obtiveram IDC menor que matrizes do GGen3, enquanto na OrdP2 não houve diferença para esta característica. Este resultado assemelhase aos de outros pesquisadores (CHANSOMBOON et al., 2010), que encontraram efeito significativo da raça da porca sobre o IDC das mesmas. Igualmente, Carregaro et al. (2006) observaram que a linhagem Camborough 22 (Large White x Landrace x Duroc) influenciou o IDC de primíparas e multíparas, de forma que o IDC diminuiu com o aumento do período lactacional das fêmeas. Os dados de Chansomboon et al. (2009) evidenciam que primíparas possuem maior IDC que multíparas, resultado que está de acordo com o encontrado no GGen3, no presente trabalho. Mellagi et al. (2013) afirmam que primíparas apresentam maior IDC no parto subsequente do que multíparas (de segunda a quinta parições), fato não encontrado no presente estudo, embora só tenham sido avaliadas matrizes de primeira e segunda OrdP.

$\mathrm{Na}$ OrdP1, fêmeas do GGen3 tiveram menor MDNP em relação aos demais e, no entanto, na OrdP2, o desempenho foi similar para esta característica. De forma semelhante ao presente trabalho, vários pesquisadores (CHANSOMBOON et al., 2009) encontraram diferença significativa para MDNP, ao avaliarem a ordem de parto das fêmeas, uma vez que, à medida que aumentou a OrdP, ocorreu uma redução na média de dias não produtivos das fêmeas.

O GPLeit diferiu significativamente em função do GGen, mas não em decorrência da OrdP da matriz. Matrizes do GGen3 produziram leitegadas com melhor desempenho para ganho médio de peso diário que as demais. A OrdP influenciou significativamente a variação no GPLeit, no experimento de Lima et al. (2006), sendo que o maior valor foi observado em leitegadas de terceiro parto. Porém, no presente trabalho não houve efeito da OrdP no ganho de peso da leitegada, considerando-se que tenham sido avaliadas apenas duas ordens de parto.

O NSPC não foi afetado pela OrdP, mas foi influenciado pelo GGen, em que fêmeas pertencentes ao GGen3 apresentaram melhor desempenho para esta característica. Semelhantemente, Giacomeli et al. (2010), ao trabalharem com matrizes Large White e Landrace cruzadas, encontraram influência da raça sobre o NSPC das mesmas. Entretanto, Ncubeet al. (2003), não verificaram efeito da raça Large White sobre o NSPC, como também das três OrdP avaliadas, resultados que corroboram os achados do presente trabalho, onde a OrdP não influenciou o NSPC das matrizes suínas. Em contrapartida, Chimonyo (2005) verificou influência da ordem de parto no NSPC da fêmea, sendo que os melhores resultados estavam em porcas de quarta e quinta ordens de parição.

Os GGen e as OrdP das matrizes não interagiram entre si e não afetaram o 
Rev. Bras. Saúde Prod. Anim., Salvador, v.16, n.1, p.47-56 jan./mar., $2015 \quad$ http://www.rbspa.ufba.br ISSN 15199940

PMNasc. Entretanto, outros autores afirmaram haver efeito da raça da matriz no peso dos leitões e da leitegada ao nascer (NOCERA \& FEDALTO, 2002; FRAGA et al., 2007). E, efeito da ordem de parto da fêmea no peso da leitegada ao nascimento (NOCERA \& FEDALTO, 2002; LIMA et al., 2006). No entanto, fatores associados ao manejo da granja avaliada, como o reagrupamento e a homogeneização das leitegadas podem ter interferido na variável PMNasc do presente estudo.

Os GGen e as OrdP das matrizes não interagiram entre si e não afetaram o TNasc e TDesm. No entanto, em matrizes Large White e Landrace cruzadas, Giacomeli et al. (2010) encontraram efeito da raça sobre o número de leitões nascidos e o tamanho da leitegada à desmama (CHIMONYO et al., 2008). Estudos tem relatado que a OrdP da matriz tem influência sobre o TNasc e TDesm (PINHEIRO et al., 2000; NOCERA \& FEDALTO, 2002; LIMA et al., 2006; GIACOMELI et al., 2010; RAZMAITE et al., 2012). Contudo, essa resposta não foi observada no presente trabalho. Ribeiro et al. (2008) encontraram diferença significativa para $\mathrm{o}$ número de leitões nascidos em fêmeas de quarta, quinta e sexta ordens de parto. Segundo Vesseur et al. (1994), há um aumento linear do tamanho da leitegada do primeiro ao sétimo partos, embora alguns rebanhos apresentem queda do número de leitões nascidos no segundo parto (AMARAL FILHA et al., 2007). Estes resultados divergem dos encontrados no presente estudo, onde matrizes de primeira e segunda lactação tiveram desempenho semelhante para a característica TNasc; este comportamento pode ter sido influenciado, em maior ou menor escala, por fatores associados ao manejo, à nutrição, ao meio ambiente e à sazonalidade.

Não houve interação nem efeito principal do GGen e da OrdP da fêmea sobre o número de leitões D/F/A. Entretanto, outros resultados demonstraram influência da raça sobre esta característica (LIMA et al., 2006; VICENTE et al., 2007; GOMES et al., 2010).

O grupo genético e a ordem de parto influenciam característicasprodutivas e reprodutivas da matriz suína. O GGen 3, resultante do cruzamento entre as raças Large White $\mathrm{x}$ Landrace, pode ser considerado superior, uma vez que apresentou melhor desempenho que os demais na maioria das características avaliadas. Não foi possível determinar a melhor ordem de parto, pois esta variou em função dos grupos genéticos e das variáveis analisadas.

\section{AGRADECIMENTOS}

À Cooperativa Agrícola São Gabriel do Oeste (COOASGO) pelo apoio e fornecimento de todo o material necessário para o levantamento $e$ tabulação de dados, como também, à empresa AGRINESS pelo fornecimento do software de gerenciamento de granjas.

\section{REFERÊNCIAS}

AMARAL FILHA, W.S.; COSTA, M.S.; MESQUITA, R.C.T.; WENTZ, I.; BORTOLOZZO, F.P. Estratégias ao desmame das primíparas para um bom desempenho subseqüente. Acta Scientiae Veterinariae, v.35, p.72-82, 2007.

BIANCHI, I.; DESCHAMPS, J.C.; LUCIA JUNIOR, T.; CORRÊA, M.N.; VARELA JUNIOR, A.S.; FONTINELLI, 
Rev. Bras. Saúde Prod. Anim., Salvador, v.16, n.1, p.47-56 jan./mar., $2015 \quad$ http://www.rbspa.ufba.br ISSN 15199940

http://dx.doi.org/10.1590/S1519-99402015000100006

É.; MEINCKE, W. Fatores de risco associados ao desempenho de fêmeas suínas de primeiro e segundo partos durante a lactação. Revista Brasileira de Agrociência, v.12, n.3, p.351-355, 2006.

CARREGARO, F.B.; MELLAGI, A.P.G.; BERNARDI, M.L.; WENTZ, I.; BORTOLOZZO, F.P. Reflexo do período de lactação na produtividade de porcas primíparas e multíparas. Acta Scientiae Veterinary, v.34, p.39-43, 2006.

CHANSOMBOON, C.; ELZO, M.A.; SUWANASOPEE, T.;

KOONAWOOTRITTRIRON, S. Genetic and Environmental Factors Affecting Weaning-to-First Service Interval in a Landrace-Large White Swine Population in Northern Thailand. Kasetsart Journal (Natural Science), v.43, p.669-679, 2009.

CHANSOMBOON, C.; ELZO, M.A.; SUWANASOPEE, T.; KOONAWOOTRITTRIRON, S.

Estimation of Genetic Parameters and Trends for Weaning-to-first Service Interval and Litter Traits in a Commercial Landrace-Large White Swine Population in Northern Thailand. Journal of Animal Science, v.23, n.5, p.543-555, 2010.

CHIMONYO, M. Evaluation of the Production and Genetic Potential of Indigenous Mukota and their Crosses with Large White Pigs in Zimbabwe. 2005. 130p. PhD (Thesis) - University of Zimbabwe, Zimbabwe.

CHIMONYO, M.; DZAMA, K.; BHEBHE, E. Genetic determination of mothering ability and piglet growth in indigenous Mukota sows of Zimbabwe. Livestock Science, v.113, p.74-80, 2008.
FÁVERO, J.A.; FIGUEIREDO, E.A.P. Evolução do melhoramento genético de suínos no Brasil. Revista Ceres, v.56, p.420-427, 2009.

FRAGA, A.B.; AZEVEDO, A.P.; SILVA, F.L.; SANTANA, R.S.; MACHADO, D.F.B.P.; COSTA, P.P.S. Peso médio do leitão, peso e tamanho de leitegada, natimortalidade e mortalidade em suínos no Estado de Alagoas. Revista Brasileira de Saúde e Produção Animal [online], v.8, p.354-363, 2007.

GIACOMELI, A.B.M.; KOZICKI, L.E.; CARVALHO, N.S. Emprego de diferentes protocolos de inseminação artificial (IA) em suínos das raças Landrace e Large White. Revista Acadêmica de Ciências Agrárias e Ambientais, v.8, p.333-339, 2010.

GOMES, S.M.A.; BERTO, D.A.; RAMOS, A.A; ORSI, R.O.

Levantamento dos dados coletados da granja de suínos da Faculdade de Medicina Veterinária e Zootecnia da UNESP. 1. Tamanho da leitegada. Veterinária e Zootecnia, v.17, p.259266, 2010.

INSTITUTO BRASILEIRO DE GEOGRAFIA E ESTATÍSTICA - IBGE. Economia. 2011. Disponível em: $<$ http://www.ibge.gov.br/home/mapa_site /mapa_site.php\#economia $>$. Acesso em: 06 jun. 2013.

KOKETSU, Y.; DIAL, G.D. Factors influencing the postweaning reproductive performance of sows on commercial farms.Theriogenology, v.47, p.14451461, 1997. 
Rev. Bras. Saúde Prod. Anim., Salvador, v.16, n.1, p.47-56 jan./mar., $2015 \quad$ http://www.rbspa.ufba.br ISSN 15199940

http://dx.doi.org/10.1590/S1519-99402015000100006

LIMA, K.R.S.; FERREIRA, A.S.; DONZELES, J.L.; MANNO, M.C.; ARAÚJO, D.; ROSTAGNO, H.S.; SILVA, F.C.O Desempenho de porcas alimentadas durante a gestação, do primeiro ao terceiro parto, com rações com diferentes níveis de proteína bruta. Revista Brasileira de Zootecnia, v.35, p.1999-2006, 2006.

MARTINS, T.D.D.; COSTA, A.N.; SILVA, H.V.; BRASIL, L.H.A.; VALENÇA, R.M.B.; SOUZA, N.M. Produção e composição do leite de porcas híbridas mantidas em ambiente quente. Ciência Rural, v.37, p.1079-1083, 2007.

MELLAGI, A.P.G.; PANZARDI, A.; BIERHALS, T.; GHELLER, N.B.; BERNARDI, M.L.; WENTZ, I.;

BORTOLOZZO, F.P. Efeito da ordem de parto e da perda de peso durante a lactação no desempenho reprodutivo subsequente de matrizes suínas. Arquivo Brasileiro de Medicina Veterinária e Zootecnia, v.65, p.819-825, 2013.

NCUBE, M.; DZAMA, K.; CHIMONYO, M.; KANENGONI, A.; HAMUDIKUWANDA, H. Effect of boar genotype on reproductive performance of the local sows of Zimbabwe. Livestock Research for Rural Development, v.15, 11p., 2003.

NOCERA, P.R.; FEDALTO, L.M. A influência de fatores ambientais e de inseminação artificial sobre as características produtivas de suínos. Archives of Veterinary Science, v.7, p.159-172, 2002.
PINHEIRO, M.J.P.; GALVÃO, R.J.D.; BARBOSA NETO, F.; ESPINDOLA, G.B. Características reprodutivas de suínos puros na região semi-árida do Rio Grande do Norte. I Tamanho da leitegada. Revista Caatinga, v.13, p.19-26, 2000.

PIRES, A.V.; LOPES, P.S.; GUIMARÃES, S.E.F.; GUIMARÃES, C.T.; PEIXOTO, J.O. Mapeamento de locos de características quantitativas associados à composição de carcaça, no cromossomo seis de suíno. Arquivo Brasileiro de Medicina Veterinária e Zootecnia, v.60, p.725-732, 2008.

RAZMAITE, V.; JATKAUSKIENE, V.; JUOZAITIENE, V. Prolificacy of old genotype Lithuanian white sows in small closed population. Acta Veterinaria, v.62, p.355-363, 2012.

RIBEIRO, J.C.; CARVALHO, L.E.; SOUSA, K.C.; NEPOMUCENO, R.C. Prolificidade de fêmeas suínas na cidade de Fortaleza, Ceará, Brasil. Archivos de Zootecnia, v.57, p.537-540, 2008.

STATISTICAL ANALYSES SYSTEM. SAS/STAT User's guide: statistics. Version 9.1. Cary, NC: SAS Institute, 5123p. 2004.

VARGAS, A.J.; BERNARDI, M.L.; BORTOLOZZO, F.P.; MELLAGI, A.P.; WENTZ, I. Factors associated with return to estrus in first service swine females.

Preventive Veterinary Medicine, v.89, p.75-80, 2009.

VESSEUR, P.C.; KEMP, B.; HARTOG, L.A.The effect of weaning to estrous interval on litter size, live born piglets and farrowing rate in sows.Journal of Animal Physiology and Nutrition, v.71, p.30-38, 1994. 
Rev. Bras. Saúde Prod. Anim., Salvador, v.16, n.1, p.47-56 jan./mar., $2015 \quad$ http://www.rbspa.ufba.br ISSN 15199940

VICENTE, A.; CAROLINO, N.;

PEREIRA L.; GAMA, L.T. Parâmetros

genéticos e efeitos ambientais de

caracteres reprodutivos em suínos

Malhado de Alcobaça. Archivos de

Zootecnia, v.56, p.655-658, 2007.

WAEHNER, M.; BRUESSOW, K.P.

Biological potentials of fecundity of sows

Zuchtungskunde. Biotechnology in

Animal Husbandry, v.25, p.523-533,

2009.

YANG, H.; PETTIGREW, J.E.;

JOHNSTON, L.J.; SHURSON, G.C.;

WALKER, R.D. Lactational and

subsequent reproductive responses of

lactating sows to dietary lysine (protein)

concentration. Journal of Animal

Science, v.78, p.348-357, 2000.

ZAR, J.H. Bioestatistical analysis. 5th

ed. New Jersey: Prentice-Hall, 2010.

944p.

Data de recebimento: 19/05/2014

Data de aprovação: 04/03/2015 